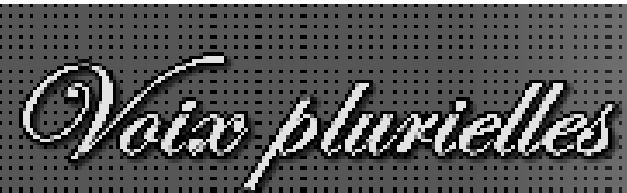

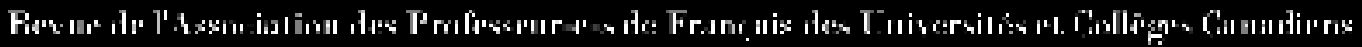

Voix plurielles

Volume 1, numéro 1 : mars, 2004

Irène Oore

Les lettres chinoises de Ying Chen: un roman épistolaire

Citation MLA : Oore, Irène. «Les lettres chinoises de ying chen: un roman épistolaire.» Voix plurielles 1.1 (mars 2004). 


\title{
Les lettres chinoises de Ying Chen: un roman épistolaire
}

\author{
Irène Oore \\ Université Dalhousie
}

Mai 2004

Ying Chen publie Les Lettres chinoises chez Leméac en 1993. Six ans plus tard, en 1999, paraît toujours chez Leméac, une seconde version, considérablement remaniée. Notre étude porte sur la version remaniée, mais nous entendons discuter brièvement du remaniement lui-même.

Le roman s'ouvre sur une lettre écrite par Yuan à sa fiancée Sassa. Yuan se trouve à Vancouver entre deux vols. La deuxième lettre du roman est également écrite par Yuan et adressé toujours à sa fiancée qu'il a quittée à Shanghai, mais cette lettre est déjà écrite de Montréal, la destination du voyage de Yuan. Ainsi donc, dès le début, Sassa est la fiancée « abandonnée », laissée derrière, une femme immobile et qui « attend», alors que Yuan est celui qui se déplace, qui quitte et qui va vers l'ailleurs. Dès lors l'échange des lettres se fera entre Shanghai et Montréal d'une façon régulière : l'un écrit, l'autre lit puis répond, chaque lettre constituant un maillon dans une chaîne, chaque personnage étant sujet écrivant et destinataire à tour de rôle.

Dès la 6e lettre nous apprenons que $\mathrm{Da} \mathrm{Li}$, une amie de Sassa, elle aussi quitte Shanghai et arrivera bientôt à Montréal. La 8e lettre est déjà écrite par Da Li à Montréal et envoyée à Sassa. Dorénavant les lettres écrites par Da Li à Sassa et celles de Sassa à Da Li s'intercaleront entre les lettres échangées par Sassa et Yuan. D'ailleurs, le lecteur a des raisons de croire que Yuan et Da Li ont une liaison et qu'ainsi la structure en mosaïque des lettres ne fait que refléter la réalité; il s'agit là de deux correspondances séparées, chacune étant dialogale mais où l'organisation séquentielle de l'échange principal entre Sassa et Yuan subit les interférences (à l'image du couple, et venant de la même source). Dans la 50e lettre $\mathrm{Da}$ Li annonce son départ imminent vers Paris. Les six dernières lettres du roman, seront, une fois de plus constituées par le duo Sassa-Yuan. C'est Sassa qui écrira la toute dernière lettre, mettant fin à la correspondance et rompant les liens avec Yuan. Une véritable symétrie structurale émerge:

\begin{tabular}{|c|c|c|}
\hline $\begin{array}{l}\text { lettres 1-7 } \\
\text { DUO }\end{array}$ & $\begin{array}{c}\text { lettres } 8-50 \\
\text { TRIO } \\
\text { S }\end{array}$ & $\begin{array}{c}\text { lettres } 51-57 \\
\text { DUO }\end{array}$ \\
\hline $\mathrm{Y}-\mathrm{S}$ & $\begin{array}{l}\mathrm{DL} Y \\
\mathrm{I}\end{array}$ & $\mathrm{Y}-\mathrm{S}$ \\
\hline $\begin{array}{l}\text { (part et initie la } \\
\text { correspondance) }\end{array}$ & $\begin{array}{l}\text { (arrive, bouleverse, } \\
\text { repart) }\end{array}$ & $\begin{array}{c}\text { (rompt la corre- } \\
\text { spondance et meurt?) }\end{array}$ \\
\hline
\end{tabular}


Nous nous proposons ici de cerner et d'examiner quelques aspects de cette correspondance fictionnelle qui en font un roman épistolaire. D'abord nous y examinerons l'intervalle spatiotemporel, l'écriture et la lecture inscrites dans tout échange épistolaire ainsi que les paradigmes de présence-absence, de révélation-dissimulation et de confrontation-clôture. Ensuite nous nous pencherons sur les choix opérés lors du remaniement de la première version du roman et sur les conséquences de ces choix.

$* * *$

La distance spatio-temporelle qui caractérise la relation sujet écrivant-destinataire constitue une donnée fondamentale de cette correspondance fictive. Dans Les Lettres chinoises la distance spatiale qui sépare Yuan de Sassa ne pourrait être plus grande; la moitié de la circonférence du globe sépare Montréal de Shanghai. Le décalage horaire est de 12 heures et le rythme du jour et de la nuit des fiancés s'en trouve diamétralement opposé (le présent diurne de l'un correspond au présent nocturne de l'autre : lorsque l'un se lève pour commencer sa journée, l'autre se couche pour la nuit).[1] L'intervalle spatio-temporel est d'autant plus important que les lettres sont expédiées par la poste et mettent au moins une semaine (sinon plus) pour parvenir à leur destinataire. Notons encore que le calendrier solaire grégorien auquel on se réfère à Montréal ne correspond guère au calendrier soli-lunaire chinois. La conversion d'une date d'un calendrier vers l'autre n'est point simple. Ceci pourrait être l'une des nombreuses raisons pour lesquelles les lettres ne sont pas datées.[2]

La distance géographique entre Montréal et Shanghai, le décalage horaire, l'intervalle temporel entre le moment de l'écriture et le moment de la lecture d'une lettre, tous ces divers intervalles s'ajoutent pour constituer une immense séparation entre Yuan et Sassa (et entre Sassa et $\mathrm{Da} \mathrm{Li}$ ), entre le « je » qui écrit et le « tu » auquel il/elle s'adresse et qui lira la lettre que le « je » écrit. Cette séparation et les conséquences qu'elle entraîne sont dramatisées davantage par la juxtaposition de la situation des deux couples; les intervalles spatio-temporels entre Yuan et Sassa et la proximité tant spatiale que temporelle entre Yuan et Da Li (qui habitent le même immeuble à Montréal) contribuent au dynamisme des échanges épistolaires ainsi qu'à celui des rapports entre les personnages.

$* * *$

La lettre reconnaît les distances, les écarts, les intervalles et tout en les reconnaissant, elle se donne pour tâche de les effacer. Il s'agit d'une re-création à travers l'écriture et à travers la lecture de l'être aimé, tellement présent dans son absence. Afin de faire survenir Yuan, Sassa raconte son désir de sa présence et ce qui pourrait combler ce désir : «Oh que j’ai besoin de toi, de tes bras et de ta poitrine pour me soutenir, et de tes baisers pour m'oublier » (LC, 26). Ailleurs, elle évoque le premier baiser qu'elle et Yuan ont échangé, un baiser au parfum de la glace aux haricots rouges : «Depuis ce jour-là, chaque fois que je pense à toi, même en plein hiver, le goût étrange de la glace amère sucrée me revient aux lèvres... » (LC, 89). Mais ces évocations, ces incantations n'arrivent pas à combler le vide laissé derrière lui par Yuan. Et Sassa, beaucoup plus que Yuan finit par capituler devant cette absence qui aura le dessus. Elle explique : « Sans toi, ma vie ne peut plus être la même. Le soleil me semble moins lumineux, et la journée trop longue » (LC, 18). C'est 
que pour Sassa le temps est ce qui la sépare de Yuan.[3] Vers la fin, Sassa reconnaît sa perte et l'absence irrévocable qu'elle entraîne : « Je t'ai perdu, toi. Oui, je t'ai perdu par un certain aprèsmidi de dimanche à l'aéroport de Shanghai » (LC, 100). Contrairement à Sassa, Yuan, lui, évoque avec plaisir un moment privilégié du passé et anticipe avec joie les retrouvailles avec sa fiancée. À travers ses lettres il surmonte l'absence : il se souvient du moment où il a pris les photos de Sassa, photos qu'il garde dans sa chambre. Il répète le prénom de Sassa, et cette simple répétition le remplit de tendresse et de bonheur : " Chaque fois que je prononce 'Sassa', je pense à la chaleur du sable, à la sonorité de ses soupirs, à la gaieté des pieds nus, à la folie du vent, à l'éternité du soleil et de la mer » (LC, 55). Pour Yuan le temps est non pas ce qui le sépare de Sassa, mais au contraire, ce qui le rapproche du moment rêvé des retrouvailles (LC, 34). Ainsi, les lettres que Sassa et Yuan échangent reflètent leur rapport aisé ou dysphorique au temps et illuminent tantôt la réalité de l'absence, tantôt l'illusion de la présence de l'autre, les deux versants de la séparation, qui est l'événement central du roman épistolaire Les Lettres chinoises.

$* * *$

Dans une de ses premières lettres à Yuan, Sassa rappelle à son fiancé un jeu du temps où ils étaient ensemble à Shanghai. Examinons pendant un moment ce jeu bien curieux qui semblait plaire beaucoup plus à Yuan qu'à Sassa. Sassa raconte :

Quand nous nous promenions tous les deux dans la rue, tu t'amusais à me lâcher soudain la main pour aller te cacher parmi la foule. Mais je t'apercevais tout de suite à travers d'innombrables têtes. Tu revenais alors auprès de moi avec le sourire las d'un prisonnier heureux. Et moi je n'aimais pas ce jeu. (LC, 32)

Ce jeu auquel Yuan s'adonnait, était-il une manifestation du désir de la liberté qu'il éprouvait? Était-il déjà une répétition pour son futur départ? Quoi qu'il en soit, ce jeu sur la séparation et les retrouvailles, sur l'illusion de l'absence et la réalité de la présence, dramatisait en filigrane et en l'inversant (en image spéculaire) le drame à venir. Mais ce jeu de Yuan était avant tout un jeu de dissimulation et de révélation. Or, l'intrigue entière des Lettres chinoises repose sur ce jeu : les lettres cachent et révèlent à la fois. C'est dans l'équilibre délicat de ce qui est caché d'une part et de ce qui est dévoilé d'autre part que se trouve le nœud de l'action.

Les confidences entre les deux femmes Sassa et Da Li se multiplient. Dès la lettre \#10 Sassa révèle à $\mathrm{Da}$ Li son état de santé alarmant $(\mathrm{LC}, 28)$. Mais elle n'en parle guère à Yuan et supplie $\mathrm{Da} \mathrm{Li}$ de ne pas le lui révéler non plus (LC, 62; LC, 118). Da Li, de son côté, révèle à Sassa son amour pour un homme, mais sans « [...] le nommer ni le décrire. Le nom et l'apparence ne sont pas importants » $(\mathrm{LC}, 51)$. Or, l'identité de cet homme est cruciale pour le développement de l'intrigue : Nous soupçonnons qu'il s'agit de Yuan; chaque lettre de Da Li ajoute de nouveaux détails sur l'homme qu'elle aime.[4] Tous ces détails confirment nos soupçons, mais $\mathrm{Da}$ Li ne révèlera pas le nom de son amant.

Nous croyons que Sassa le soupçonne, mais tout en le suggérant, elle ne le dit jamais d'une façon explicite et directe. Ainsi, raconte-t-elle à Yuan l'histoire d'une femme « [...] qui, à force 
d'attendre son mari séparé d'elle par une large rivière, est devenue une pierre [...] » $(\mathrm{LC}, 59)$ puis enchaîne en interpellant Yuan : "Tu sais, plusieurs soupçonnent que la rivière ne soit pas le véritable obstacle pour ce couple, mais bien le cœur de l'amoureux en question » (LC, 59). Se doute-t-elle déjà de l'infidélité de son fiancé?

De par sa nature monologale, la lettre est un instrument parfaitement adapté à la révélation ainsi qu'à la dissimulation. Si l'acte de lecture de la lettre est un moment décisif de reconnaissance, dans Les Lettres chinoises la lettre qui en résulte n'est jamais un acte ouvert de confrontation. Plutôt il s'agit, une fois de plus, d'un acte voilé mi-révélation, mi-dissimulation. Chacun des trois personnages écrit ce genre de lettres « obliques », lettres qui font entendre, qu'il ou elle en sait bien plus qu'il ou elle ne dit. Ces lettres sont souvent doublement ironiques. Sassa écrit à Da Li à propos de la liaison de celle-ci : «Je ne sais pas pourquoi ton affaire m'excite à ce point, comme s'il s'agissait de mon propre bonheur » (LC, 73). L'ironie est qu'il s'agit bel et bien du bonheur de Sassa et que vraisemblablement elle le reconnaît. Da Li écrit à son tour à Sassa et admet : "Chaque fois que je pense à toi, j'ai envie de pleurer. Comment vont les choses pour toi? » (LC, 110). Cette envie de pleurer, n'est-elle pas l'admission d'une culpabilité profonde? Et la sollicitude de la question qui suit « Comment vont les choses pour toi » semble d'autant plus suspecte.

Enfin Yuan demande pardon à Sassa en s'accusant de fautes tellement abstraites qu'elles semblent négligeables, il s'accuse de s'être trompé sur la place des droits et des devoirs, mais n'admet jamais la véritable raison pour laquelle il devrait demander pardon. " Et surtout, je te demande pardon si parfois je perds pied malgré moi dans ce mélange de devoirs et de droits, et que je me trompe sur ma place à leur accorder au fond de moi » (LC, 116). Et dans son cynisme agressif Yuan supplie et insiste : « Tu me pardonnes, n’est-ce pas? Dis-moi que tu me pardonnes, Sassa! » (LC, 116).

Le roman épistolaire permet de cette façon aux demi-vérités ou demi-mensonges de créer et de maintenir l'ambiguïté de l'intrigue. Or cette ambiguïté est un ingrédient essentiel pour le suspense du récit.

$* * *$

L'échange des lettres que sont Les Lettres chinoises est précipité par les départs de Yuan puis de $\mathrm{Da}$ Li de Shanghai à Montréal. Qu'est ce qui met fin à cet échange? En apparence c'est le départ de Da Li de Montréal à Paris qui interrompt (momentanément?) sa correspondance avec Sassa. En réalité n'est-ce pas sa rupture de la liaison avec Yuan qui fait qu'elle n'a plus besoin de la confidente qu'est devenue Sassa? Et si toute la correspondance était un jeu pervers de destruction, le but n'est-il pas atteint?

La correspondance entre Sassa et Yuan se termine-t-elle parce que Sassa est trop malade pour écrire, ou encore, parce que l'infidélité de Yuan mène vers une rupture des fiançailles? Enfin, on est en droit de se demander si les relations entre Sassa et Yuan n'étaient pas condamnées d'avance, car Yuan tenait à quitter la Chine alors que Sassa voulait rester à Shanghai. Toutes ces questions restent ouvertes à la fin du roman. Dans une tradition caractéristique au genre épistolaire Les Lettres chinoises choisissent l'ouverture et l'ambiguïté à la clôture.

Avant de terminer cette étude des Lettres chinoises et de leur inscription dans le genre du roman épistolaire, nous aimerions examiner le remaniement important du roman pour la deuxième 
édition de l'ouvrage. Cet examen pourra, nous l'espérons, illuminer davantage certaines questions portant sur l'esthétique épistolaire.

La première version des Lettres chinoises (Leméac, 1993) contient 69 lettres, 4 sujets écrivants et 4 destinataires. Cette version a 171 pages. La deuxième version abrégée et modifiée (Leméac, 1999) contient 57 lettres (donc 12 lettres ont été supprimées), 3 sujets écrivants, mais assez curieusement toujours 4 destinataires. Cette deuxième version a seulement 132 pages, donc une quarantaine de pages est supprimée. Plusieurs lettres sans être éliminées ont été considérablement abrégées.

Dans la première version, Sassa et le père de Yuan écrivent de Shanghai à Montréal, où se trouvent Yuan le fiancé de Sassa qui correspond avec son père et avec Sassa et Da Li qui échange des lettres avec Sassa. Dans la deuxième version, les lettres du père sont supprimées et une seule lettre de Yuan à son père reste (il y en avait 7 et une lettre a changé de destinataire).

La première version comporte une certaine symétrie au niveau des sujets écrivants et des destinataires :

- un homme et une femme à Shanghai

- un homme et une femme à Montréal

- les fiancés Sassa à Shanghai et Yuan à Montréal écrivaient à 2 destinataires chacun, un homme et une femme.

Cette symétrie disparaît de la deuxième version où Sassa écrit à Yuan, son fiancé et à son amie $\mathrm{Da} \mathrm{Li}$ qui est aussi sa rivale. Yuan, quant à lui, écrit uniquement à Sassa, sauf dans un cas où une lettre s'adresse à son père. Le résultat de la suppression de toutes les lettres écrites par le père de Yuan et de la plupart de celles écrites par Yuan à son père, est que des trois sujets écrivants qui restent, Sassa écrit le plus grand nombre de lettres : 27 lettres sont de Sassa, 20 lettres sont de Yuan et 10 lettres sont écrites par Da Li.

Puisque Yuan et Sassa sont fiancés et puisqu'il semble que Yuan trompe Sassa avec son amie $\mathrm{Da} \mathrm{Li}$, la 2e version du roman est plus intense. Le père de Yuan, ne faisant pas partie du triangle amoureux, constituait un élément non-passionnel, un élément de détente dans la première version. Dans la deuxième version il est à peine présent et reste silencieux comme le destinataire d'une seule lettre. La raison pour préserver cette unique lettre (lettre \#37 dans la première version devenue lettre \#29 dans la deuxième version) n'est guère évidente.[5] La lettre bien qu'intéressante ne semble guère indispensable. De plus parce qu'elle devient unique, son importance grandit : or était-ce l'intention? Nous en doutons. En fait, la supprimer aurait été du point de vue esthétique un choix plus cohérent. Le roman épistolaire aurait eu trois correspondants représentant le triangle amoureux

Une autre décision prise lors du remaniement nous laisse perplexes : il s'agit de la lettre \#5 de Yuan qui était dans la première version adressée à son père et qui dans la deuxième version se trouve abrégée et adressée à Sassa. Le contenu de cette lettre traite des bains (à Shanghai et à Montréal), du désir de Yuan de rassurer sa mère qu'il est content : le changement du destinataire ne se fait point ici sans une maladresse remarquable.[6] Nous nous demandons, une fois de plus pourquoi ce changement du destinataire, et surtout pourquoi préserver à tout prix cette lettre?

De plus plusieurs lettres de la première version sont considérablement abrégées dans la seconde. Il s'agit surtout des lettres de Yuan qui se trouvent plus brèves dans la deuxième version. 
Il en résulte (comme avec la suppression de la voix du père) une plus grande intensité, une plus grande urgence. De plus, le personnage de Yuan semble plus énigmatique, ce qui met en relief l'ambivalence et le suspense de l'intrigue. Nous proposerions en guise de conclusion une troisième édition du roman où le personnage du père disparaîtrait entièrement (en tant que destinataire) et la lettre \#5 serait supprimée. Une nouvelle symétrie triangulaire apparaîtrait alors et le roman s'en trouverait à la fois plus dépouillé et plus équilibré.

Les deux duos de voix du début et de la fin, et le trio du milieu atteindraient ainsi une plus grande pureté. L'échange des lettres mènerait inéluctablement, tragiquement vers le silence ultime de la rupture et de la mort.

\section{NOTES}

[1] Voir à ce propos la lettre \#4 et l'image du soleil et de la lune qu'évoque Yuan dans cette lettre. Il y écrit à Sassa : « Fais attention, ma belle lune, tu risques de te brûler en embrassant ton soleil. Mais il a tellement besoin de toi. Tu es sa seule source d'énergie. S'il se lève tous les jours, c'est dans l'espoir de te revoir. Pourquoi doit-on attendre cette lueur de crépuscule pour pouvoir se rencontrer $[\ldots] »(\mathrm{LC}, \mathrm{LC}, 15)$

[2] Alors que certains indices temporels (références au temps qu'il fait à Montréal et à Shanghai, références à des fêtes chinoises ou montréalaises) nous permettent de déduire qu'entre la première lettre de Yuan (au mois de janvier) et la lettre d'adieu de Sassa (octobre? novembre?) s'écoulent dix à onze mois, alors que ces références nous permettent de situer approximativement chaque lettre par rapport aux saisons et parfois par rapport au mois, il nous est impossible de parler du rythme des envois, c'est-à-dire des ralentissements ou des accélérations qui ont lieu.

[3] Dans une autre lettre Sassa note : «A cause de ton absence peut-être [...] [j] ne m'accroche plus très bien à mon quotidien. Je glisse ». (LC, LC, 26)

[4] L'homme vient de Shanghai, il a une fiancée à Shanghai et il attend qu'elle vienne à Montréal. Il habite dans le même immeuble que Da Li.

[5] La lettre se trouve dans la deuxième version au milieu du volume, intercalée entre une lettre de Da Li à Sassa et une autre de Sassa à Da Li. Elle hausse un peu le suspense. Dans cette lettre Yuan explique à son père qu'il est heureux à Montréal et réfléchit sur la faillibilité de la nature humaine.

[6] Voir pages 16 et 17 de la nouvelle édition. 